\title{
Exploration and Thoughts on the Status Quo of Chinese Opera Stage Art
}

\author{
Ying Zheng ${ }^{1,2, *}$
}

\author{
${ }^{1}$ Yunnan Arts University, Kunming, Yunnan 650050, China \\ ${ }^{2}$ Moscow State University, Lomonosov, Russia \\ *Corresponding author. Email: 340074782@qq.com
}

\begin{abstract}
From 2005 to 2020, Chinese opera stage art, based on inheritance and innovation, adopts all kinds of advanced technologies, uses Chinese and foreign aesthetic concepts for reference, attracts audiences in more professional and diverse forms, and brings them excellent visual enjoyment. This article takes the stage art creation of opera from 2005 to 2020 as the research object. Through 15 years of case analysis of modern stage art to grasp the development status of modern stage art, this article explores the dilemma of modern stage art creation, further studies the development prospects of stage art, and starts thinking about the development trend of stage art in Chinese opera.
\end{abstract}

\section{Keywords: Chinese opera, stage art, development trend, innovative development, analysis of new works}

\section{INTRODUCTION}

In recent years, based on inheritance and innovation, the stage art of Chinese opera has adopted various advanced technologies and borrowed from Chinese and foreign aesthetic concepts, attracting audiences in more professional and diverse forms, and bringing them excellent visual enjoyment. The prospect of Chinese opera is great. Not only has the government attached great importance to it, it has also received a large number of special fiscal expenditures and policy support. President Xi Jinping has also advocated the innovative development of traditional culture on many occasions. In 2017, the state promulgated the "Opinions of the Central Government on Implementing the Inheritance and Development Project of Chinese Excellent Traditional Culture" and other policies, which have achieved a good reputation and momentum in the society. The stage art of Chinese opera has also made great strides. Especially in the rehearsal of old operas, its stage design has been newly improved in line with the trend of the times, bringing the audience a visual enjoyment of keeping up with the times. At present, the stage design of opera shows a state of "inheritance" and "innovation", and it has been reformed with modern new trends and Western aesthetic methods on the basis of inheriting traditional Chinese aesthetics. As an indispensable part of opera, stage art enables the audience to understand the origin of opera stories and the important visual language of emotion, which is an intuitive factor that can attract audiences. Through the case analysis of modern opera stage art and the mastery of the development status, this article explores the difficulties in the creation of modern stage art, further studies the development prospects of stage art, and develops thinking about the development characteristics of Chinese opera stage art and the future trend, so as to better promote the overall development of Chinese opera.

\section{THE TRANSFORMATION CHARACTERISTICS OF MODERN OPERA STAGE}

Due to the lack of materials in traditional operas, most of them focus on "freehand brushwork", with only "one table and two chairs" or various fabrics such as tents as simple decorations, which is unavoidably monotonous. But nowadays, the stage art has undergone earth-shaking changes. Various elements are ingeniously integrated, and the level of coordination between stage scenery and plot and theme development is also increasing, creating a perfect professional visual image. Just like in the Chinese opera stage art design that Chinese accomplished stage artist Mr. Liu Xinglin has participated in over the years, whether it is the Kun Opera version of "Confucius's Into the Wei" (2016) or "Lu You and Tang Wan" (2019), He always insisted on the integration of "material" and "freehand brushwork". On the basis of "one table and two chairs", he combined garden elements and at the same time cooperated with lighting to further develop the freehand brushwork and hypothetical nature of the opera stage art. With incorporating things of diverse nature and drawing upon the strengths of others, he makes the Chinese opera stage embark on an international perspective, giving Chinese opera a place in the world stage art design. Chinese opera is well-known in the world. After comprehensively analyzing the major operas in recent 
years, it can be concluded that: on the basis of new concepts, new technologies and the use of new materials, on the premise of inheriting the model of "one table and two chairs", the stage art setting has produced a completely personalized reform, and the stage design also shows more modern aesthetics.

\section{A. A variety of setting methods}

After the continuous innovation of opera stage art, the stage is no longer limited to a single element, but begins to adapt to the overall stage setting, becoming a converged, unified and coordinated stage. It pays more attention to creating scenery based on emotions, highlighting the integrity of the opera stage. For example, in Peking Opera "Red Cliff" (2008), whether it is the canopy on the stage or the patterns on the curtain, they are all based on the characteristics and expression of the era of the Three Kingdoms Period. In the Yue Opera "Early Spring in February" (2018), simple props are used, for example, a few bamboos with white banners represent the cemetery, and reed cloth and chairs represent the dilapidated home. It uses simple but typical features to express the environment, so as to cooperate with the actors, form an organic whole with the plot and the characters' situation, and increase the integrity of the opera on stage.

On this basis, modern stage art has added new technologies such as sound, light, electricity and other forms of expression to highlight the environment and increase the degree of integration and visual effects. For example: "The New Madam White Snake" (2009) also used the projection screen technology to bring the scenery of West Lake to the stage, which greatly increased the real sense of the scene, and the weather changes in the projection even highlighted the complex emotional changes of the white snake. In Bai Xianyong's version of "The Story of the Jade Hairpin" (2014), advanced screen casting technology is also used to complete the scene transformation to express the inner ups and downs of the characters. In the Kun Opera youth version "Peony Pavilion" (2019), a large number of projections are used, and in the first backdrop, Chinese calligraphy is used to create a large background of scholarly family. In the plot "Sweet Dream in the Garden", dreamlike colors are projected on the backdrop, creating a colorful garden scene in the dream.

In addition to projection, an important innovation in modern stage art is the use of film visual effects as the setting. The peculiarities of opera performances make all the performances of the actors must be done in one go without any mistakes. However, with the continuous development of opera stage art and the emergence of various new media, many operas have also begun to add film elements to stage art to increase the appreciation and sense of hierarchy of stage art. For example, the Peking Opera "Red Cliff" (2008) uses the "montage" commonly used in movies, that is, a site with multiple scenes. However, it is not only performing synchronously on the same stage, but also using lighting or other means to produce an intuitive experience of scene visual switching. In the second act of "Red Cliff", when Sun Quan discussed the strategy with the ministers, while Sun Quan was furious, the characters on the stage fell into a standstill. At this time, the stage lights illuminated the veil curtain of paintings in Han Dynasty, and the scene of another high platform (that is, the Bronze Peacock Terrace) began to appear, and $\mathrm{Cao} \mathrm{Cao}$ intoned on it. After the singing, the lights dimmed, and the scene setting was switched back to the Sun Quan scene again. At this time, a scene transition was completed. It cleverly uses the lighting and the scene in scene to create a transition effect like a movie screen, giving the audience a feeling of spatial transformation. In addition to "montage", in terms of expression techniques, the film effect combining virtual and real is also widely used in the opera stage. For example, the pre-recorded video of Peking Opera "The New Madam White Snake" is shown on the screen, creating a dreamlike sense of flying from the sky to the man's world, achieving the intersection of virtual and reality.

There is also a trend of using new materials to make settings, such as creating fairyland scenes through dry ice, and using special effects to create natural scenes. In Kun Opera "1699.Peach Blossom Fan" (2006), new mirror reflective materials were used to create the feeling of the Qinhuai River water surface. It seemed that the whole scene was on the river, making the whole stage more open and magnificent, showing the lively cities along the Qinhuai River to the fullest.

In addition, there is a newest popular display mode, namely the interactive mode of opera and video. In 2018, "Inner Awareness" created by Feng Qianhui used a new model to interpret the scene of "Sweet Dream in the Garden" in "Peony Pavilion". It uses modern new technology such as projection, kinect body recognition, computer human-computer interaction, etc., so that the audience can walk freely in the veil curtain, observe the real-time performance from all angles, and have an immersive and wonderful experience. It reinterprets the definition among audience, actors and drama stage, giving opera stage art a new development form and direction.

\section{B. It is compatible, open and constantly deepening}

Compared with traditional stage art expression, modern stage art is more open and flexible. It has a little less restraint and restriction, and a more compatible attitude of advancing with the times. Stage art is considered and improved based on understanding and following the spiritual needs and aesthetic level of 
modern audiences. After integrating the strengths of many schools of thought, the stage of opera has greater tension in artistic performance, and its appeal is better transmitted. For example, the youth version of Kun Opera "Peony Pavilion" (2019), adapted by Bai Xianyong and performed abroad, made its debut in the Netherlands with an amazing innovation. Based on the aesthetic needs of modern audiences, this play is shortened to $80 \%$ of the original play on the basis of the original version, which further highlights the aesthetic characteristics of Kun Opera by simplifying it by cutting out the superfluous, and at the same time it is more in line with the pace of life and needs of young audiences. In the "Peony Pavilion" in the Netherlands, in order to allow the Dutch to understand the meaning of the plot more and appreciate the charm of lyrics, in this stage art of "Peony Pavilion", it cooperated with the Dutch modern dance regiment for the first time to use a pair of local male and female dancers for creative performance. In "Peony Pavilion", with the development of the hero's love, the lingering feelings of emotion are expressed through the body dance with full of tension, which makes the audience be infected by the delicacy and elegance of Kun Opera. The cello was also added for the first time in the composition to increase the penetrating power and appeal of the melody. And with the use of projection digital equipment, it makes the stage integrated with the fresh and freehand elegant colors of Chinese ink painting, and the traditional calligraphy and traditional Chinese painting are designed on the stage. The whole stage still retains Chinese characteristics after adding modern dance elements.

The combination of foreign dance and the combination of Chinese and Western performances has greatly shortened the distance between the Dutch audience and the traditional Chinese Kun Opera, and has formed a connection between the audience and the opera stage performances, which not only reduces the audiences' aesthetic fatigue, but also gives audiences an unprecedented sensory experience. The association of activity and inertia and the combination of rigidity and softness gave Western audiences a different spiritual feeling for the artistic conception of Kun Opera. Obviously, this kind of performance finally achieved great success.

\section{Independent and professional stage art team}

Mr. Gong Hede mentioned in the requirements of the opera stage in "Speaking of Peking Opera" (2016) that: "Make the stage performance a distinct artistic whole that can arouse appreciation from the audience". And an important way to meet this requirement is to build a well-organized, efficient and professional stage art team in the opera team.
For a long time, the stage performance of Chinese opera was very simple and crude. However, with the continuous improvement of stage art technology, the scope of stage aesthetics continues to increase, and the contradictions between various stages and components have begun to intensify. What follows is the need for a professional team to be fully responsible for the stage art presentation of each play, propose complete design ideas and master all aspects of the stage. In 2018, the Grand National Theatre "Taihu Stage Art Center", which integrates stage art design and production, technical exchange, prop manufacturing and art education, was officially put into use. This is also a large-scale and fully-functional formal stage art institution second to none in China and foreign countries, which means that stage art will enter a new stage of development.

Therefore, there are more and more professionals in modern opera stage art, and the number of institutions and teams specializing in stage art research is also increasing. Under the direction of the director's overall artistic conception, each performs its own duties, and through mutual cooperation and coordination, jointly creates and arouses the audience's appreciation of the opera stage art.

\section{REASONS FOR THE TRANSFORMATION OF MODERN OPERA STAGE}

The stage art of Chinese opera is constantly changing in an era, changing in tradition, and has an extremely distinctive contemporary character. And the modern opera stage has become more various, diversified and open in the continuous development of realistic writing and freehand brushwork, and has taken a path of its own.

\section{A. The development and changes of opera itself}

Chinese opera itself has become somewhat dull and boring to modern audiences as the era has changed. It is even said that certain values and concepts are different from modern values to a certain extent, and these differences have also led to the continuous reform of opera. At the same time, along with the continuous integration of foreign cultures from the West, the opera gradually began to transform from within itself. The importance of stage art, as an existence that accompanies opera, has gradually been seen by more and more people. Therefore, the development of opera will inevitably lead to changes in stage art. For example, "True Man" (2010) performed on the capital stage is an obvious play with spoof elements, which has been accepted and widely praised by young people, which also represents the spread of the mashup style of opera. In its stage art design, whether it is atmosphere or music, designer Sun Daqing chose a design full of Beijing flavor, and specially built a carriage that can 
move on the stage, thereby increasing the overall sense of the stage. On the basis of not losing authenticity, the advantages of many drama stages are integrated into the opera stage design, and the scene and drama are perfectly integrated.

The new version of Peking Opera "Tang Concubines" (2019) also conforms to the propositions in the current large-scale drama discussion, transforming "modernization" into "dramatization". Adhering to the insistence of "seeing the new in the old and having roots in the new", "Tang Concubines" was re-arranged, and after removing the pattern elements, it returned to the opera itself. What followed was the stage art design that closely followed the changes of this Peking Opera. The new version of the design paid more attention to details in the costumes, props, and makeup of each character, focusing on highlighting the style of Tang Dynasty. Decorated tables and chairs with richer texture and contemporary flavor were adopted, which made the whole stage more exquisite and has the beauty of the prosperity of the Tang Dynasty.

\section{B. Variety of audience aesthetic forms}

Due to the overall improvement of the aesthetic level of modern audiences, the immutable old skills are no longer able to satisfy the audience's requirements, and the traditional and simple stage can't attract the audience's attention. Various emerging art forms continue to impact people's lives. Different forms of communication media and dizzying sound and light have also stimulated the improvement of people's aesthetic standards and broadened their horizons. The aesthetic needs have gradually begun to develop to a higher level. In the traditional sense, "one table and two chairs" will naturally appear monotonous, dull and boring. Therefore, it is an inevitable trend for stage art designers to use various methods to enrich stage scenes.

\section{Renewal and iteration of technological means}

The modern opera stage has a rich and diverse update of technological means. Today's opera stage has a more accurate grasp of the "degree" of stage technology, so that stage technology and opera have reached a complete harmony, and the audience's overall viewing experience is improved. For example, in "The New Madam White Snake" (2009) of the Peking Opera, in the scene of the white snake's "descending", the use of splendid lighting colors creates a virtual reality method, the use of lighting creates mountains and clouds, and the use of constantly changing lighting simulates the weather with dense clouds and lightning and thunder to achieve excellent visual effects. The stage in the "Chinese New Year Opera Gala" in 2012 added a sense of space to the stage through the design of three steps of ladder. An LED main screen was designed between the two columns to increase the sense of virtuality and reality. Such combination makes the stage level richer, and the design of the large-screen stage can be seen almost every year in the Chinese New Year Opera Gala.

In the 2016 G20 Summit, it was based on "The Butterfly Lovers", one of the four ancient Chinese folk love stories. The play was the world's largest holographic AR Theater performance project at the time. It used the spectra vision technology in the FantaView Magic Vision system and used the principle of optical illusion to combine the images (people and objects) shot by Musk's camera technology in the movie with the main model landscape in the setting box. It demonstrates the development process of the story according to the script, which is vivid and unpredictable, with special effects such as sound, light and electricity, which are novel and intuitive. The advantage of spectra vision is that it is not restricted by factors such as venues, can better display a certain three-dimensional sense and sense of the scene, and can also be adjusted according to the size of the venue, so that the audience can directly feel the sense of hierarchy and strong depth of the picture sense. Similarly, the huge long curtain in 2017 made the scenery of the Tibetan area in the Zang Opera "Songtsen Gambo" completely natural. In addition, in the new version of "Tang Concubines" (2019), the stage art team used the multimedia equipment of the Shanghai Grand Theater to coordinate the LED screen and the projection to replace the physical building in the original version, making the scene more magnificent and grand. In the scenes of "Huaqing Hot Spring" and "Maweipo", the stage art team used LED screens to show the tranquility and emptiness of Huaqing Hot Spring and the deep affliction in Maweipo to the right level, which is not overly rendering and can make people empathize.

\section{CONCLUSION}

Although the current stage art development is in good condition, it's also needed to take a rational view of the unevenness of stage art in various regions. Due to the economic level of various regions and the living conditions of theater companies, the development of stage art varies greatly. Regarding the development characteristics of stage art mentioned in the content of this paper, each region should rationally treat the gap in stage art development, cultivate its own strong professional qualities, and take a new look at the characteristics of local type of drama. After combining the living habits and national culture of the local people, it's necessary to carry out the corresponding stage art creation, thereby creating stage art suitable for the development of the region, injecting new vitality into stage art, and gaining the support and favor of the masses. In addition, modernity and tradition should also be taken into consideration, and the degree of integration should be grasped. 
The current stage art is developing in a colorful direction with the times. While making breakthroughs, it's also important to preserve traditions, strike a balance between innovation and tradition, adhere to traditional opera stage art concepts such as "conservation", "traditional stage", and "one table and two chairs", boldly give feedback to modern society, go deep into life for observation and experience, learn from the excellent essence of Chinese and foreign stage forms, cleverly use the combination of high-tech rationality and then create stage art, giving the stage art an endless vitality. Modern stage art presents a new and personalized look on the principle of inheriting traditional opera stage art, thus reaching a mature stage. Regardless of the form of expression, content of expression or means of use, it has reached a new height, and many outstanding stage art works have appeared. However, in terms of the grasp of "degree" and the balance of development, further exploration and research are still needed to make stage art to a higher level in terms of persistence and innovation. Chinese opera has a glorious past and should also have a brilliant future. And the Chinese opera stage should also have a splendid and colorful development prospect. The transformation of the development of opera stage reflects the focus of exploring the balance between inheritance and innovation of traditional culture in modern society. Opera goes with the times. Only by coordinating the advancement of various local operas can people create a new situation in which Chinese operas flourish. Chinese opera stage art still has a lot of room for development, waiting for people to dig and explore.

\section{References}

[1] Li Xu. Research on the Evolution of Chinese Opera Stage Scenery [D]. Jiangsu Normal University, 2013. (in Chinese)

[2] Zhou Junjie. Thirty Years of Contemporary Opera Stage Art Creation (1980-2010) [D]. Chinese Academy of Art. (in Chinese)

[3] Zhou Hui. The Directors' Exploration and Construction of the Modern Narrative Style of the Opera Stage [D]. Shanghai Theatre Academy. (in Chinese)

[4] Zhu Mengyu. Experimental Interpretation of the Stage Art of "Peony Pavilion" [D]. Nanjing University of the Arts, 2019. (in Chinese)

[5] Zheng Ying. Research on the Application of New Media Art in Theatrical Stage Design [D], Kunming University of Science and Technology, 2011. (in Chinese)

[6] Free Discussion on the Stage Art of Chinese Opera [M]. Yunnan: Yunnan Nationalities Publishing House, 2020. (in Chinese) 